\title{
Conceptualization of Perceptual Attributes: A Special Case for Color?
}

\author{
Nicola J. Pitchford and Kathy T. Mullen \\ McGill University, Montreal, Quebec, Canada
}

\begin{abstract}
Young children experience difficulties establishing conceptual representations of color compared with everyday objects. We argue that comparing the development of color cognition to that of familiar objects is inappropriate since color is a perceptual attribute that can be abstracted from an object and by itself lacks functional significance. Instead, we compared the recognition, perceptual saliency, and naming of color to that of three other perceptual object attributes (motion, form, and size) in 47 children aged 2 to 5 years as a function of language age. Results revealed that, although color was perceptually salient relative to the other visual attributes, no selective impairment to color cognition (recognition and naming) was found relative to the three other visual attributes tested. Thus, when the appropriate comparisons are made, we find no special delay in the development of color conceptualization. Furthermore, the striking disparity between perceptual saliency and cognition of color in our youngest age groups suggests that perceptual saliency has little influence on the conceptual development of color. () 2001 Academic Press

Key words: color cognition; visual development; perceptual saliency.
\end{abstract}

It has long been believed that young children have difficulty establishing conceptual representations of color, based on the striking discrepancy in their ability to learn the names for everyday objects and the tardy, erratic nature by which they appear to learn color terms (e.g., Baldwin, 1893; Cruse, 1977; Darwin, 1877, cited in Bornstein, 1985a; Modreski \& Goss, 1969; Nagel, 1906). At an early stage of color term learning, children may acquire color terms without understanding their precise reference and will often apply color terms haphazardly and inconsistently. For example, when asked about the color of an object young chil-

Supplementary material (a color version of Fig. 1) is available on IDEAL (http://www. idealibrary.com). This research was supported by Medical Research Council Project Grant MT10819 to K.T.M. We are particularly grateful to the children, staff, and directors (Ellen Unkrig Staton and Carole Montpetit, respectively) at the Royal Victoria Hospital and Montreal General Hospital daycares that kindly participated in this research. We thank Dr. Tim Ledgeway for the computer programming, Rebecca Achtman for recording the task instructions, Paul Maloney for help with an early literature review, and three anonymous reviewers for providing very helpful comments on an earlier draft.

Address correspondence and reprint requests to Nikki Pitchford, McGill Vision Research, Department of Ophthalmology, 687 Pine Avenue West, Rm. H4-14, Montreal, H3A 1A1, Quebec, Canada. E-mail: npitch@po-box.mcgill.ca. 
dren may respond with a color word, although not necessarily the correct one, and may use a single color word to name many different colors.

Accurate color naming is taken as an indicator of color cognition, as the ability to name colors accurately involves the transference of information from conceptual color-processing mechanisms to stored phonological lexical color codes (Davidoff, 1991). An established literature has suggested that accurate color naming develops surprisingly late, at around 4-7 years (e.g., Bornstein, 1985a; Heider, 1971; Johnson, 1977; Mervis, Catlin, \& Rosch, 1975), and that color terms may be learned only through explicit instruction, sometimes requiring hundreds of presentations before the appropriate term is learned (Rice, 1980).

The apparent difficulty children exhibit in learning to identify and name colors accurately is particularly striking given that their ability to perceive and discriminate color is established in infancy. Substantial psychophysical evidence suggests that infants can distinguish color differences within the first few weeks of life (e.g., Maurer \& Adams, 1987; Teller, 1998; Teller \& Bornstein, 1985); color contrast sensitivity develops in line with luminance contrast sensitivity (Allen, Banks, \& Norcia, 1993; Teller, 1998); and the basic color categories are perceived by 4 months of age (Bornstein, Kessen, \& Weiskopf, 1976). A perceptual saliency for color presumably develops later as young children of around 3 years can use color as a basis for sorting colored papers into piles (Miller \& Johnston-Laird, 1976) and grouping together objects (e.g., Baldwin, 1989; Brian \& Goodenough, 1929; Itskowitz, Strauss, \& Gross, 1987; Melkman, Koriat, \& Pardo, 1976; Suchman \& Trabasso, 1966). By around the age of 4 years, preferential-matching tasks show a shift from a preference for color to a greater saliency for form (Suchman \& Trabasso, 1966), although the precise age at which the transition occurs varies across studies (e.g., Baldwin, 1989; Miller, 1977).

Since a perceptual deficit cannot account for the discrepancy between young children's ability to learn color words and everyday object labels, the delay may lie in the development of conceptual representations of color or its referential mapping that underpin the ability to identify and name colors accurately (Bornstein, 1985b; Soja, 1994). In this sense, young children resemble adults with acquired color agnosia, who exhibit a selective impairment to color concepts (Davidoff, 1991). Bornstein (1985b) noted that both young children and adults with color agnosia make perseverative or random errors in naming colors, although they perceive color normally, and he suggested that both may reflect functional deficits in the same neurological substrate. In adults with color agnosia, acquired color naming deficits arise from damage to the cortical structures that subserve color cognition, while in children, selective difficulties in learning color names may reflect an immature state of maturation or integration of these cortical structures. Specifically, he proposed that the cortical structures that integrate visual-verbal abilities are not in place until children reach approximately 4 years of age, and only after this maturational threshold is it possible to observe accurate color naming.

Neuromaturation accounts of color naming difficulties in young children, or at least the time scale suggested, have been criticized by studies demonstrating 
experiential and cohort effects on color term knowledge (Andrick \& TagerFlusberg, 1986; Anyan \& Quillian, 1971; Bartlett, 1978; Bateman, 1915; Macario, 1991; Miller \& Johnston-Laird, 1976; Shatz, Behrend, Gelman, \& Ebeling, 1996). For example, attendance at school (Anyan \& Quillian, 1971; Bateman, 1915) or preschool (Shatz et al., 1996) has been shown to refine color term knowledge. Additionally, contemporary 2-year-olds have been shown to have better color term knowledge than children of previous generations (Shatz et al., 1996) that reportedly could name the four primary colors by 7 years in the 1900s (e.g., Binet \& Simon, 1908/1916) and 4 years in the 1970s (Miller \& Johnston-Laird, 1976). Such effects would not be expected if children were limited by a maturational threshold for learning color terms (Shatz et al., 1996).

Even though contemporary children exhibit better color term knowledge than previous generations (Shatz et al., 1996) it remains to be explained why children find color terms effortful to learn and why they appear at a later developmental stage than labels for familiar objects. Unlike color words, children learn the words for many everyday objects with apparent ease and with remarkable speed during the early stages of lexical acquisition, often within a single occurrence of hearing the word spoken in context (e.g., Carey, 1978).

The task of early word learning is considered principally referential, which involves learning how words refer to objects, actions, and events in the world (e.g., Markman, 1989; Nelson, 1991). A critical difference exists, however, in the functional significance of color compared with everyday objects that may inhibit the early acquisition of color concepts. Color is an abstract object property, by which we mean it is a perceptual attribute than can be abstracted from an object, and by itself lacks the functional significance that is inherent in objects. It has been proposed that children may initially conceptualize object properties that are functionally relevant and, compared to form, color is less informative about an object's function (Au \& Markman, 1987; Macario, 1991; Soja, 1994). For example, objects that share a common name may differ in color (e.g., a sweater may be blue, green, orange), and objects that are labeled differently may have the same color (e.g., a rose, a heart, a strawberry). In contrast, an object's function tends to be closely related to its form. Consequently, children may learn to expect linguistic labels to refer to an object's form rather than its color (Baldwin, 1989). However, for objects that are similar in form, color is a critical property for identification, so color only becomes important when drawing within-category distinctions. Accordingly, accurate color naming may only emerge when children begin to make within-category distinctions or differentiate between similarly shaped objects. Twenty-one-month-old infants show an awareness of adjectives referring to abstract object properties (such as color or texture) when objects are drawn from within the same basic level category but not when objects are drawn from between categories (e.g., Waxman \& Markow, 1998). If children's color naming difficulties arise because color is an abstract visual attribute the conceptualization of other visual attributes (such as shape or size) should be comparable to that of color. Selective delays in the cognitive development of color may not 
be observed when the conceptualization of color is compared to that of other abstract visual attributes.

Color may also differ from objects in the precision of its conceptual boundaries. Braisby and Dockrell (1999) contrasted 3- and 5-year-olds' conceptualization (comprehension and naming) of colors and animals that varied in word frequency and found that children could comprehend and name high-frequency colors (e.g., red) and animals (e.g., cow) to the same extent. In contrast, children were selectively poorer at conceptualizing low-frequency colors (e.g., aquamarine) compared to low-frequency animals (e.g., seahorse). The authors argued that the differential deficit with conceptualizing low-frequency colors relative to lowfrequency animals reflects differences in the precision of the conceptual representations of these two categories: low-frequency animal concepts have sharp boundaries, whereas low-frequency color concepts have blurred boundaries. They also predicted that children should experience similar selective difficulties with conceptualizing other imprecise visual attributes, such as size.

Given the differences in the functional significance and possibly the underlying conceptual representations of color compared to familiar objects, as outlined above, it may not be surprising that children learn to conceptualize everyday objects prior to color. We therefore suggest that claims that color naming develops surprisingly late (e.g., Bornstein, 1985a; Heider, 1971; Johnson, 1977; Mervis, Catlin, \& Rosch, 1975) need qualification as the key question seems to be: late in relation to what? We argue that it is more appropriate for studies to compare the development of color cognition to that of other abstract perceptual attributes, such as abstract forms or size, than comparing the conceptualization of color to functional objects, yet very few studies have taken this approach.

Few empirical studies have compared the naming of color and abstract forms. A study by Bornstein (1985a) compared the paired-associate learning of proper nouns to each of three different abstract forms (e.g., a square, a triangle, and a circle colored black) and three different colors (e.g., a blue, a green, and a yellow circle) in a group of children aged 3 years. The children learned significantly more shape-name associations, at a faster rate and with fewer errors, than color-name associations, leading Bornstein (1985a) to conclude that young children experience greater difficulty associating names to color than form.

In addition, a recent study by Sandhofer and Smith (1999) contrasted the learning of color and size terms in 2-year-old children. They compared six color terms (red, orange, yellow, green, blue, purple) with two size terms (big, little) in each of three tasks: a comprehension task ("Show me the red/big one"), a production task ("What color/size is this?"), and a property abstraction task ("What matches this?"). Results showed that children had more difficulty in comprehending the colors than the sizes and more difficulty matching objects on the basis of color compared to size. In addition, children appeared to have linked a set of color terms to the question "What color is this?" even though they could not comprehend the color terms they produced. On this basis, Sandhofer and Smith (1999) argued that children use a different system of multiple mappings to learn color 
words compared to size words and that the early linking of color words to color questions promotes the mapping of color terms to their referents. This contrasts directly with Braisby and Dockrell's (1999) prediction, and our own, that children should conceptualize imprecise property attributes, such as color and size, in a similar manner.

In this study we investigated the development of color cognition in comparison to three other primary perceptual attributes (form, size, and motion), which, like color, are abstract concepts lacking functional significance. We also selected these properties because they are all basic visual attributes that receive specialized processing by the visual system in the prestriate coretx (e.g., De Yeo \& Van Essen, 1988; Hadjikhani, Liu, Anders, Cavanagh, \& Tootell, 1998; Livingstone \& Hubel, 1988). Thus, we compare the conceptualization of color with motion, size, and abstract forms to determine whether the difficulties children experience in conceptualizing color genuinely reflects a selective difficulty with color or a more general delay in conceptualizing abstract object attributes.

We used young children aged below 5 years that were grouped according to language age rather than chronological age, as this should provide a more reliable metric by which to control for overall language ability. Language age was determined by a standardized psycholinguistic language assessment that included measures of both receptive and expressive language. We compared directly the conceptualization of color (red and green) to that of motion (fast and slow), size (big and small), and abstract forms ( $\mathrm{O}$ and $\mathrm{T}$ ) over three experimental tasks. A recognition task assessed children's comprehension of color compared to the other visual attributes. This task also served to establish the relative familiarity of the different stimuli used. A preferential-matching task investigated the perceptual saliency of color, relative to motion, size, and abstract forms, during the developmental period in which visual concepts are established. A confrontational naming task compared the relative ease by which children could produce the spoken names for color, form, motion, and size. Performance on both the recognition task and the confrontational naming task was taken to indicate conceptualization, since the ability to comprehend and produce a spoken word accurately requires an intact conceptual representation. If, as claimed by previous studies, color presents a special case for conceptualization, we would expect children to show a selective impairment in recognizing and naming color relative to the other abstract visual attributes. In contrast, if the delay in conceptualizing color relative to functional objects arises from their status as an abstract property attribute, we predict that children will recognize and name color with comparable accuracy to the other abstract property terms.

\section{METHOD}

\section{Participants}

Forty-seven normally developing English-speaking children from two different daycare settings participated in the study. Prior consent was obtained from 
parents or guardians. The socioeconomic status and race of the children was typical for a multicultural society such as Montreal. All children had normal vision and hearing and all but one had experienced normal language acquisition up to the time of testing, as judged by their parents. The one child in whom speech and language difficulties were reported had received therapy and his condition was considered fully remediated. The 24 boys and 23 girls were divided almost equally into four chronological age groups: $\leq 2$ years (22 to 35 months, $M=28.2$ months); 3 years (37 to 46 months, $M=41.7$ months); 4 years (48 to 59 months, $M=52.3$ months); and $\geq 5$ years ( 60 to 63 months, $M=61.3$ months). ${ }^{1}$ The children were then redistributed across the four age groups according to their language-age as assessed below.

\section{Language Assessment}

Language development was assessed using the Preschool Language Scale-3 (Zimmerman, Steiner, \& Pond, 1992). This standardized psycholinguistic scale has two subscales: (1) auditory comprehension measured receptive language abilities and (2) expressive communication assessed expressive language skills. For auditory comprehension, children were required to point to specific pictures, and for expressive communication to produce spoken answers in response to questions asked by the experimenter. The auditory comprehension subscale was administered first. Raw scores from the two subscales were summed to form a total language score, from which age-equivalent scores were determined ranging between a possible lower limit of 0 years 0 months to a possible upper limit of 7 years 0 months. The distributions of children across the four age groups according to chronological age (CA) and language age (LA) is given in Table 1. All further reference to the subject groups refers to LA groups only.

\section{Apparatus and Stimuli}

Stimuli were computer-generated 8-bit images, generated using custom software developed in our laboratory, and were presented under control of a portable computer connected to a color monitor (refresh rate of $67 \mathrm{~Hz}$ and resolution of $640 \times 480$ pixels). We selected two examples of each of the four visual attributes being investigated: red and green (color), fast and slow (motion), small and big (size), and $\mathrm{O}$ and $\mathrm{T}$ (abstract forms). Stimuli were visually clear examples of each of the four attributes that were matched, as far as possible, for familiarity (as established by the recognition pretest). As oral naming of the stimuli was required it was important that the stimulus names were of similar linguistic complexity; only stimuli with monosyllabic names that did not contain a complex cluster were selected. This excluded basic shapes, such as a square, circle, or triangle. As the

\footnotetext{
${ }^{1}$ Although the chronological age of the children in this group did not exceed 63 months, their corresponding language ages extended to 81 months; therefore, we considered it more appropriate to label this group $\geq 5$ years).
} 
TABLE 1

Distribution and Descriptive Statistics for Each of the Four Age Groups According to

Chronological Age (CA) and Language Age (LA)

\begin{tabular}{|c|c|c|c|c|c|c|c|c|}
\hline \multirow[b]{2}{*}{ LA group } & \multicolumn{4}{|c|}{ CA (months) } & \multicolumn{4}{|c|}{ LA (months) } \\
\hline & $n$ & Mean & $S D$ & Range & $n$ & Mean & $S D$ & Range \\
\hline$\leq 2$ years $(n=9)$ & 14 & 28.2 & 4.2 & $22-35$ & 9 & 29.8 & 3.8 & $23-34$ \\
\hline 3 years $(n=19)$ & 14 & 41.7 & 3.2 & $37-46$ & 19 & 41.8 & 3.3 & $37-47$ \\
\hline 4 years $(n=7)$ & 10 & 52.3 & 4.0 & $48-59$ & 7 & 52.4 & 3.1 & $48-57$ \\
\hline$\geq 5$ years $(n=12)$ & 9 & 61.3 & 1.1 & $60-63$ & 12 & 71.7 & 7.5 & $60-81$ \\
\hline
\end{tabular}

children were nonreaders ${ }^{2}$ at the time of testing, $\mathrm{O}$ and $\mathrm{T}$ were considered appropriate examples of abstract forms that lacked functional significance.

Stimuli are shown in Fig. 1 and were constructed in the following way: (1) form, $\mathrm{O}$ and $\mathrm{T}$ were drawn in uppercase; each form was 50 pixels high and 50 pixels wide $(2.5 \times 2.5 \mathrm{~cm})$, subtending $4.75^{\circ}$ of visual angle at a typical sitting distance of $30 \mathrm{~cm}$; (2) color, red was created by setting the red, green, and blue guns (of the monitor) to the values of $255,0,0$, respectively, and $0,255,0$ for green, respectively. These gun settings created colors that were judged to be good examples of red and green. Stimuli were presented against a white background created by setting the red, green, and blue guns to the same value of 255 ; (3) motion, fast and slow stimuli were created by displacing the stimulus at $13 \mathrm{~cm} / \mathrm{s}$ for fast and $3.3 \mathrm{~cm} / \mathrm{s}$ for slow. Stimuli oscillated continuously in phase horizontally at 0.9 s/cycle; (4) size, the shape stimuli (O and $\mathrm{T})$ described in (1) were used for the small size and were magnified by 2 to create the big size.

\section{Procedure}

Each child was tested individually by the first author in a quiet area of the daycare. A daycare educator was present during the testing of some of the younger children. The child sat on a chair facing the monitor at a distance that enabled the child to touch the screen easily. The experimenter explained to the child that they were going to play some games on the computer. Each task started with some lively music and the appearance of a cartoon monkey called "Charlie." Task instructions, spoken by a female, were recorded and presented via "Charlie." After the music, "Charlie" introduced the game by saying, "Hi. My name's Charlie and we're gonna play a game. Are you ready?" The first trial was presented (specific details are given in the subsequent sections). The stimuli remained on the screen until the child responded. A short beep after the child's response had been entered into the computer marked the end of each trial, and the

${ }^{2}$ Children were considered nonreaders as they had not received any formal reading instruction at the time of testing. This is of importance because for nonreaders the letter stimuli lacked functional significance and were thus conceptually abstract. We address the relevance of this issue further under Discussion. 
(a)

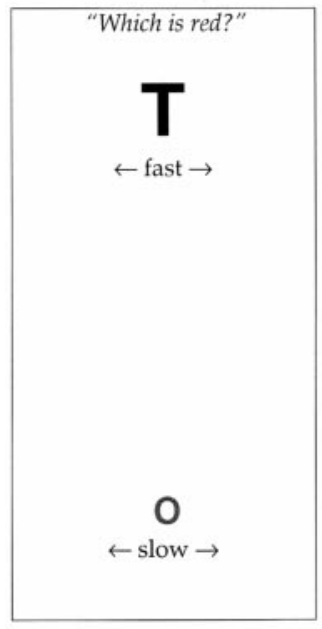

(b)

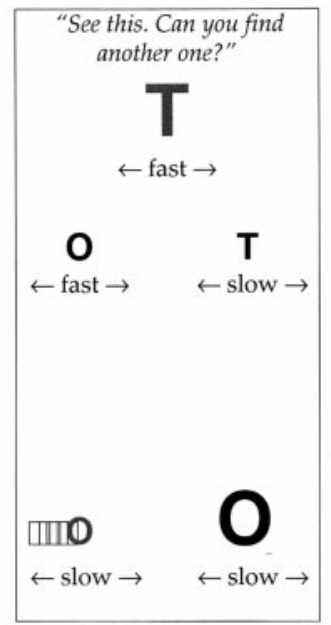

(c)

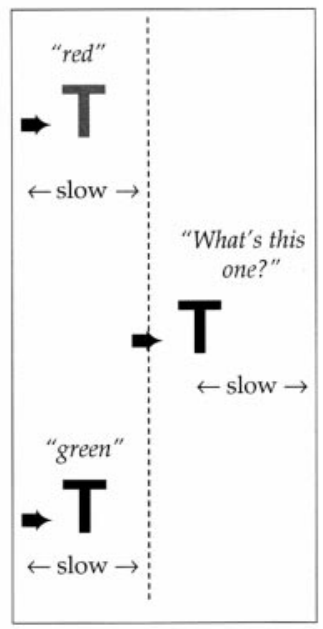

\section{Key: $\mathbf{w}=$ red $\quad \boldsymbol{a}=$ green}

FIG. 1. Schematic illustration of the stimuli display used in: (a) the recognition task; (b) the preferential-matching task; and (c) the attribute naming task. In (c), the attribute naming task, the stimulus pair was presented simultaneously to the left of the screen (as indicated by the dashed line) and remained on the screen as the target was presented to the right of the stimulus pair. The dashed line was not part of the stimulus display. In all tasks, stimuli remained on the screen as the target was presented and the child had made his or her response. The illustrated stimulus displays corresponds to (a) trial 1 of the recognition task (see Appendix 1); (b) trial 9 of the preferential-matching task (see Appendix 2); and (c) color stimulus pair 6 of the attribute naming task (see Appendix 3).

following trial was presented. Blocks of four trials were interspersed with short cartoon movies that played for approximately $30 \mathrm{~s}$ to maintain the child's attention. During the movie encouraging comments were given, such as "keep going" and "yippee." The end of the game was indicated by the music that played at the start and the appearance of "Charlie," who said, "Thanks for playing my game. You were very good." Each child completed the tasks over several sessions lasting approximately $10 \mathrm{~min}$ each. Three tasks were administered.

(1) Recognition task. A short test for recognition of the four visual attributes was given first (pretest) and again after (posttest) each child had completed the matching and naming tasks. This task also served to establish the relative familiarity of the eight different stimuli used. A pair of stimuli, differing across each of the four visual attributes, were presented simultaneously in the center of the screen, one above the other (see Fig. 1a); for example, if the first stimulus was T, red, fast, and big the second stimulus was $\mathrm{O}$, green, slow, and small. The eight possible stimulus pairs were each presented once in random order (see Appendix 1 for the full set of stimuli). On each trial the child was required to point to one of the two stimuli presented on the screen in response to a spoken word (e.g., 
"red"). The position of the target stimulus (top or bottom) was randomized over trials. Each example of the four visual attributes (red, green, fast, slow, big, small, $\mathrm{O}, \mathrm{T}$ ) was presented once for recognition, producing a total recognition score of 2 for each of the four visual attributes investigated (color, motion, size, and form).

(2) Preferential-matching task. The preferential-matching task was designed to establish the relative perceptual saliency of the four visual attributes for children of different language ages and was administered before the confrontational naming task. The task required the child to find a match to a target stimulus by pointing to one of four alternative forced choices (see Fig. 1b). The target stimulus represented the four visual attributes (e.g., T, red, fast, and big). Each of the four possible matches to it had one attribute in common with the target stimulus, but differed over the remaining three attributes (e.g., if the target stimulus was T, red, fast, and big the stimulus that matched in form would be T, green, slow, and small). The stimuli (red, green, fast, slow, big, small, O, T) of the four visual attributes (color, motion, size, and form) can be combined into 16 different targets that were randomly presented over one block of 16 trials (see Appendix 2 for the full set of stimuli). Two blocks of 16 trials were presented, producing a total of 32 trials.

At the start of each trial a target stimulus appeared at the center top of the screen and the child was asked the question "See this. Can you find another one?" This question was used by Baldwin (1989) and allowed children to match on the basis of either color or form. Accordingly, we used this question to enable children to match on the basis of any of the four visual attributes. The target stimulus was presented for $5 \mathrm{~s}$, after which the four choices simultaneously appeared below the target (as shown in Fig. 1b). The position of the four alternative forced choices was randomized across trials to control for position response biases. All of the stimuli remained on the screen until the child indicated his or her preference match by pointing to one of the four choices.

(3) Attribute naming task. A confrontational naming task was given to assess the relative naming of the four visual attributes by children with different language ages. The child was required to produce the spoken name for each of the two examples of the four visual attributes. The eight stimuli were presented in pairs that differed only in the attribute to be named (see Appendix 3 for the full set of stimuli). At the start of each trial, a stimulus pair was presented simultaneously (as shown in Fig. 1c). A small arrow pointed to each stimulus in turn, while the computer voice-over named the particular stimulus (e.g., "red" and "green"). Each stimulus was named twice and remained on the screen while the target stimulus appeared in the middle-right of the screen. The arrow then pointed to the target stimulus and after a short interval ( $3 \mathrm{~s})$ the voice-over asked the child, "What's this one?" All of the stimuli remained on the screen until the child had made his or her response. The position of the target attribute was randomized across trials, as was the order of targets to be named. Each stimulus was presented eight times for oral naming, producing a total of 64 trials (16 per visual attribute).

Responses were recorded as either correct or incorrect, with a correct response considered the name given to each of the eight different stimuli at pretest (e.g., 
"red" for the red stimuli). Only the stimulus labels used in the pretest were produced by the children in the naming task, with the exception of "little" for the stimulus labeled small, which we accepted as correct. Although different types of errors were possible (for example, within-category errors, e.g., "blue" for a red stimulus; between-category errors, e.g., "big" for a red stimulus; and omissions), all errors were simply recorded as incorrect. When a child provided a between-category response (for example, "big" when shown a big, fast, red, T on a trial contrasting color) the trial was repeated to highlight the attribute being questioned. If the child continued to provide an attribute name from a different category than the one being questioned his or her response was recorded as incorrect.

\section{RESULTS}

\section{Recognition Task}

This task assessed the children's comprehension of the four visual attributes, prior to (pretest) and after (posttest) the matching and naming tasks. Mean performances for each of the LA groups on this task are shown in Table 2. As can be seen, by a language age of 4 years the children were performing mostly at ceiling. Differences in mean performance of the four LA groups at pre- and posttest across the four visual attributes were explored by a $4 \times 2 \times 4$ mixed ANOVA, where LA group was the between-subjects factor and test session and visual attribute were the within-subjects factors. Significant main effects of LA group, $F(3,43)=15.29, p<.0001$, and visual attribute, $F(3,129)=3.72, p<.05$, were found. The main effect of test session was not significant and neither were any of the interactions. Posthoc analyses (Tukey-Kramer, $p<.05$ ) showed (1) the $\leq 2$ years LA group were significantly poorer at comprehending the visual attributes than children with a language age of 3 years and above and (2) motion was comprehended significantly less than color and size. These results suggest that children's comprehension of visual attributes follows a typical developmental progression as children with higher language ages outperformed those of a younger language age. They also suggest that three of the visual attributes, color, size, and abstract forms, could be comprehended to the same extent by the children within each LA group, even the youngest one. Furthermore, the failure to find a main

TABLE 2

Mean Performance (of Two) on the Recognition Task for Each LA group at Both Pre- and Posttest

\begin{tabular}{|c|c|c|c|c|c|c|c|c|}
\hline \multirow{3}{*}{$\begin{array}{l}\text { LA group } \\
\text { (years) }\end{array}$} & \multicolumn{8}{|c|}{ Visual attribute mean $(S D)$ of two } \\
\hline & \multicolumn{2}{|c|}{ Color } & \multicolumn{2}{|c|}{ Form } & \multicolumn{2}{|c|}{ Motion } & \multicolumn{2}{|c|}{ Size } \\
\hline & Pre & Post & Pre & Post & Pre & Post & Pre & Post \\
\hline$\leq 2(n=9)$ & $1.4(0.7)$ & $1.2(0.7)$ & $1.0(0.9)$ & $1.1(0.9)$ & $1.3(0.5)$ & $1.1(0.8)$ & $1.1(0.9)$ & $1.6(0.5)$ \\
\hline $3(n=19)$ & $1.9(0.2)$ & $1.8(0.5)$ & $1.7(0.6)$ & $1.6(0.6)$ & $1.4(0.6)$ & $1.7(0.5)$ & $1.9(0.3)$ & $2.0(0.0)$ \\
\hline $4(n=7)$ & $2.0(0.0)$ & $2.0(0.0)$ & $2.0(0.0)$ & $2.0(0.0)$ & $1.6(0.8)$ & $2.0(0.0)$ & $2.0(0.0)$ & $2.0(0.0)$ \\
\hline$\geq 5(n=12)$ & $2.0(0.0)$ & $2.0(0.0)$ & $2.0(0.0)$ & $2.0(0.0)$ & $1.8(0.6)$ & $2.0(0.0)$ & $2.0(0.0)$ & $2.0(0.0)$ \\
\hline
\end{tabular}


effect of test session indicates that the matching and naming tasks had not influenced the children's comprehension of the visual attributes, suggesting that no effect of learning had occurred over the course of the experiment.

The task also served to establish the relative familiarity of the eight different stimuli used prior to the matching and naming tasks. A $4 \times 8$ mixed ANOVA (where LA group was the between-subjects factor and stimuli was the within-subjects factor) revealed a significant main effect of LA group, $F(3,43)=12.53$, $p<.0001$, and stimuli, $F(3,301)=2.98, p<.001$, though the interaction was not significant. Posthoc analyses showed that only one of the eight stimuli was recognized to a lesser extent: children comprehended slow significantly less than red, green, fast, big, and small (Tukey-Kramer, $p<.05$ ). Importantly, these results suggest that (with the exception of slow) the stimuli were matched for familiarity. Consequently, any differences in the children's ability to match or name the different stimuli cannot be attributed to differences in their ability to comprehend the stimuli, as all but one of the stimuli were comprehended to the same extent.

\section{Preferential-Matching Task}

This task assessed the relative perceptual saliency of the four visual attributes for children at different points of language development. The results for the preferential-matching task for each of the four LA groups are reported in Table 3 and Figs. 2 and 3. As the scores for the visual attributes on this particular task were dependent on each other it was not possible to perform a mixed ANOVA on the data, as the assumptions of ANOVA would be violated. Consequently, three separate analyses were performed on the data to explore both the between- and within-subject variation: (1) Differences in the mean performances between LA groups for each of the visual attributes were investigated using four between-subjects ANOVAs. Significant effects of LA group were found for each visual attribute (color, $F(3,43)=10.12, p<.0001$; motion, $F(3,43)=10.30, p<.0001$; size, $F(3,43)=10.30, p<.001$; and form, $F(3,43)=21.53, p<.0001)$. Posthoc analyses (Fisher's PLSD test, $p<.05$ ) were conducted to explore further the differences between LA groups, the results of which are reported in the appropriate sections below; (2) the pattern of performance across visual attributes with-

TABLE 3

Mean Number of Visual Attributes Selected (of 32 Trials) on the Preferential-Matching Task for Each LA Group

\begin{tabular}{|c|c|c|c|c|}
\hline \multirow{2}{*}{$\begin{array}{l}\text { LA group } \\
\text { (years) }\end{array}$} & \multicolumn{4}{|c|}{ Visual attribute mean $(S D)$ of 32} \\
\hline & Color & Form & Motion & Size \\
\hline$\leq 2(n=9)$ & $8.6 \quad(4.5)$ & $12.3(7.3)$ & $5.9(3.4)$ & $5.2(2.4)$ \\
\hline $3(n=19)$ & $17.7(11.0)$ & $8.4(8.7)$ & $2.7(2.9)$ & $3.1(3.6)$ \\
\hline $4(n=7)$ & $0.9 \quad(0.7)$ & $29.3(2.9)$ & $0.9(0.9)$ & $1.0(1.8)$ \\
\hline$\geq 5(n=12)$ & $3.2(9.2)$ & $28.3(9.3)$ & $0.3(0.9)$ & $0.3(0.9)$ \\
\hline
\end{tabular}




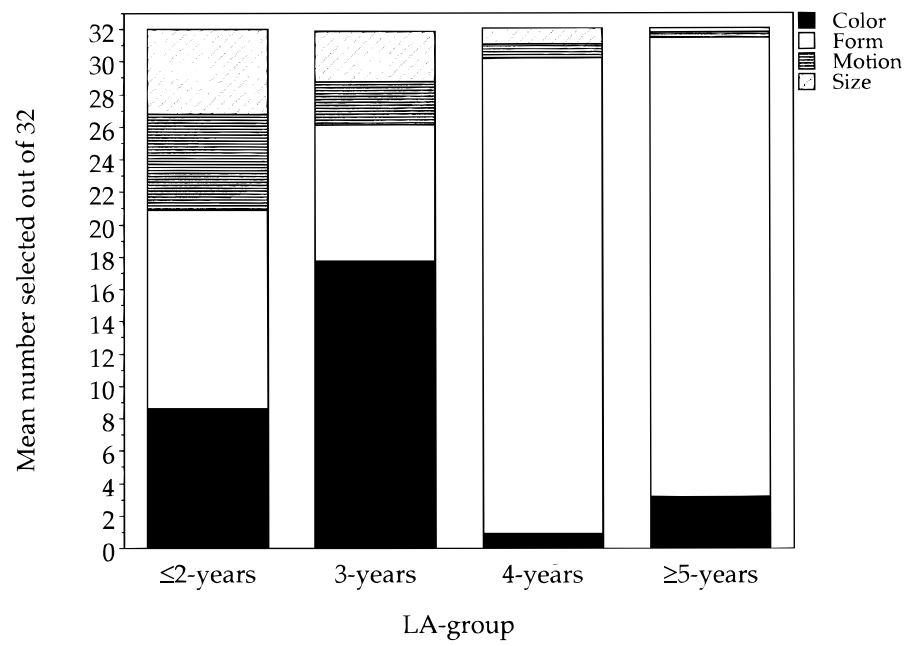

FIG. 2. Mean performance (number selected of 32 trials) for each of the four language age (LA) groups on the preferential-matching task.

in each of the LA groups was investigated using one-group $\chi^{2}$; the total number of times an attribute was chosen by a particular LA group was compared against the expected frequency of that attribute being selected by chance. Results were shown in Fig. 2; (3) the distribution of individual children exhibiting a preference for a particular visual attribute within each LA group was investigated by conducting one-group $\chi^{2}$ on individual children's data. When $\chi^{2}$ yielded a significant

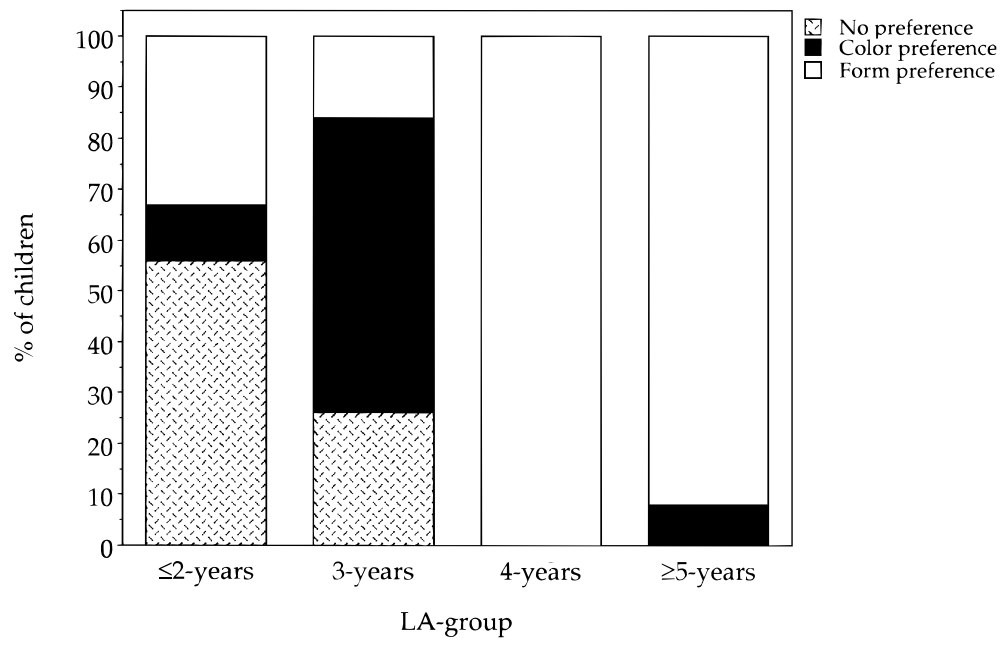

FIG. 3. Proportion of children (\%) in each of the four language age (LA) groups exhibiting a particular preference behavior on the preferential-matching task. 
result the child was considered to show a preference for that attribute. If a null result was obtained the child was categorized as showing "no preference." Accordingly, children fell into one of three categories: no preference, color preference, and form preference. Results are shown in Fig. 3. We found that none of the children showed a preference for motion or size. To see if the distribution of children exhibiting a preference for either color or form differed from chance, the data for each LA group were subjected to a one-group $\chi^{2}$. The results from these analyses are summarized below for each LA group.

Two years and under. A mixed pattern of results was found for the children in the $\leq 2$ years LA group. The within-group analyses (Fig. 2) revealed that this group matched significantly more on the basis of form than the other visual attributes, $\chi^{2}=35.17, p<.0001$. In total they made 111 form matches, 77 color matches, 53 motion matches, and 47 size matches (expected frequency, 72). However, the individual preference behavior analysis revealed that most of the children in this group, $56 \%$ (5/9), showed no preference for a particular visual attribute, while $33 \%$ (3/9) exhibited a form preference and $11 \%$ (1/9) expressed a preference for color (Fig. 3). The proportion of children exhibiting a preference for form compared to color did not differ significantly from chance, $\chi^{2}=1.0, p=.32$.

Three years. A reasonably consistent pattern of preference behavior was found for the children with a language age of 3 years. Posthoc analyses from the between-group ANOVAs showed these children made significantly more preference matches based on color than each of the other LA groups, $p<.01$ (Fig. 2). Within this group, children made significantly more matches on the basis of color than form, motion, and size, $\chi^{2}=348.28, p<.0001$. A total of 160 form matches, 337 color matches, 52 motion matches, and 59 size matches (expected frequency, 152) were made. The analysis of individual preferences (Fig. 3) showed that more than half of the children with a language age of 3 years, 58\% (11/19), had a preference for color, whereas $26 \%$ (5/19) had no preference and $16 \%(3 / 19)$ had a preference for form. Significantly more children in this group expressed a preference for color than form, $\chi^{2}=4.57, p=.03$.

Four years. A very clear pattern of results was found for children with a language age of 4 years. Posthoc analyses of the between-group ANOVAs showed (Fig. 2), that they made significantly more preference matches on the basis of form than children with a younger language age, $p<.0001$. Furthermore, within this group, significantly more form-based preference matches were made relative to those based on color, motion, and size, $\chi^{2}=528.61, p<.0001$. In total, children in the 4 years LA group made 205 form matches, 6 color matches, 6 motion matches, and 7 size matches (expected frequency, 56), and all of the children in this group showed a significant preference for form, $\chi^{2}=7.0, p=.01$ (Fig. 3).

Five years and above. A clear pattern of results was found for children in the $\geq 5$ years LA group. Posthoc analyses from the between-group ANOVAs showed (Fig. 2) that they matched significantly more on the basis of form than children with language ages less than 3 years, $p<.0001$. Also, within this group children made significantly more form-based preference matches than those based on 
color, motion, and size, $\chi^{2}=835.40, p<.0001$. Overall, children with a language age of $\geq 5$ years made 340 matches on the basis of form, 38 on the basis of color, 3 on the basis of motion, and 3 on the basis of size (expected frequency, 96). Furthermore, 92\% (11/12) of the children showed a preference for form and 1 child, $8 \%$, showed a preference for color (Fig. 3), a distribution that differed significantly from chance, $\chi^{2}=8.33, p<.01$.

Overall, these results reflect a developmental trend in the perceptual saliency of visual attributes for young children. Children that expressed no preference behavior had younger language ages $(M=2: 11$ years, $S D=4.33$ months) than those preferring color ( $M=3: 8$ years, $S D=8.86$ months). Likewise, children showing a color preference had younger language ages than those preferring form ( $M=4: 8$ years, $S D=16.84$ months). Differences between the mean language ages of these groups of children were found to be significant using a betweensubjects ANOVA, $F(2,44)=11.20, p<.0001$, and the form-preference group had significantly higher language ages than those exhibiting a color preference or no preference (Fisher's PLSD, $p<.05$ ).

\section{Attribute Naming Task}

The aim of this task was to assess the relative naming ability of the four visual attributes by children with different language ages. Each of the eight stimuli (red, green, fast, slow, big, small, O, T) were presented for naming on eight trials. The naming scores for the two stimuli representing each of the four visual attributes (color, motion, size, and form) were combined to produce a total naming score of 16 for each attribute. Table 4 and Fig. 4 report mean naming scores for each of the four visual attributes on the confrontational naming task for each LA group. Three analyses were performed on the data. To establish the extent to which children at different stages of language development could name the four visual attributes we compared differences in mean naming performance (Fig. 4) and differences in naming consistency (Fig. 5) for each attribute between the different LA groups. To establish the role of perceptual saliency in the establishment of attribute concepts, we compared differences in mean naming performance for each of the visual attributes between children grouped according

\section{TABLE 4}

Mean Number of Visual Attributes Named Correctly (of 16) on the Confrontational Naming Task for Each LA Group

\begin{tabular}{lrrrr}
\hline \multirow{2}{*}{$\begin{array}{l}\text { LA group } \\
\text { (years) }\end{array}$} & \multicolumn{4}{c}{ Visual attribute mean $(S D)$ of 16} \\
\cline { 2 - 5 } & \multicolumn{1}{c}{ Color } & \multicolumn{1}{c}{ Form } & Motion & Size \\
\hline$\leq 2(n=9)$ & $8.9(3.0)$ & $9.6(3.5)$ & $9.0(2.7)$ & $9.9(3.6)$ \\
$3(n=19)$ & $14.9(2.0)$ & $12.9(4.3)$ & $13.3(3.1)$ & $14.6(2.1)$ \\
$4(n=7)$ & $16.0(0.0)$ & $15.3(1.3)$ & $15.6(0.8)$ & $16.0(0.0)$ \\
$\geq 5(n=12)$ & $16.0(0.0)$ & $16.0(0.0)$ & $15.9(0.3)$ & $15.9(0.3)$ \\
\hline
\end{tabular}




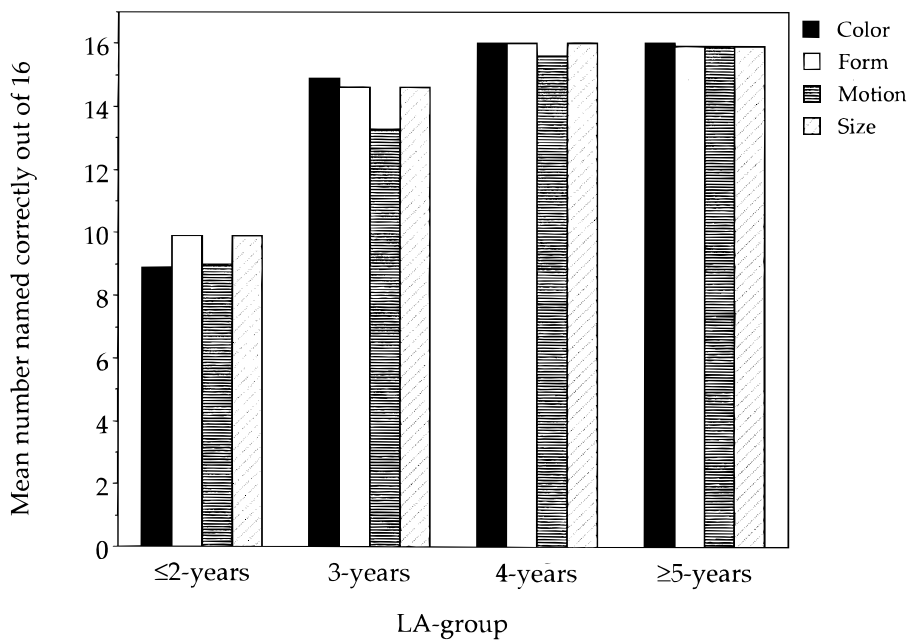

FIG. 4. Mean number of visual attributes named correctly (of 16) for each of the four language age (LA) groups.

to their particular preference behavior exhibited in the preferential-matching task.

(1) Analysis of naming accuracy in relation to language age. Differences in the mean naming performance of the different LA groups across the four visual attributes were investigated with a $4 \times 4$ mixed ANOVA, where the between-subjects factor was LA group and the within-subject factor was visual attribute. A significant main effect of LA group was found, $F(3,43)=24.59, p<.0001$.

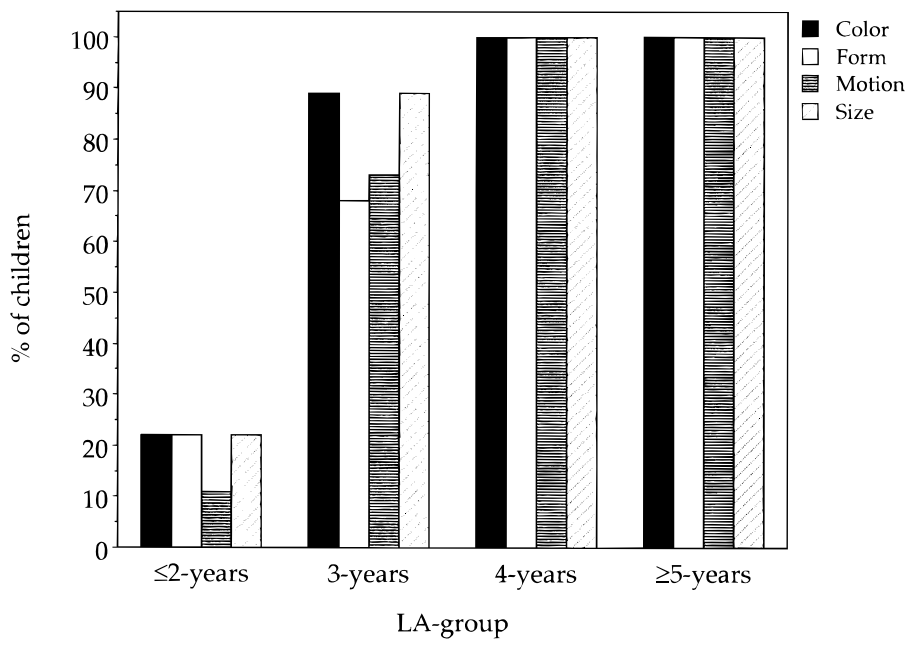

FIG. 5. Percentage of children in each of the four language age (LA) groups that consistently named the visual attributes. 
However, neither the main effect of visual attribute nor the interaction was significant (visual attribute, $F(3,129)=1.69, p=.17$; interaction, $F(9,129)=1.35$, $p=.22$ ). Posthoc analyses (Tukey-Kramer, $p<.05$ ) revealed that children with a language age of $\leq 2$ years named significantly fewer attributes than the other LA groups. Furthermore, children with a language age of 3 years were significantly poorer at naming the attributes than children with a language age of 4 years and above. There was no difference in the naming abilities of the two older LA groups as most children with a language age greater than 4 years performed at ceiling (see Fig. 4).

(2) Analysis of naming consistency in relation to language age. Children were classified as naming a visual attribute consistently if they achieved a score of $12 / 16$ or more ( $p<.05$, one-tailed binomial test). Results are shown in Fig. 5. For the $\leq 2$ years LA group, form was named consistently by $22 \%$ (2/9), color by $22 \%$ (2/9), motion by $11 \%(1 / 9)$, and size by $22 \%(2 / 9)$. The 3 years LA group named form consistently by $68 \%$ (13/19), color by $89 \%$ (17/19), motion by $73 \%(14 / 19)$, and size by $89 \%$ (17/19). All of the children in the 4 years and $\geq 5$ years LA groups named the four visual attributes consistently.

Differences in mean naming consistency between the LA groups across the four visual attributes were investigated by subjecting the data to a $4 \times 4$ mixed ANOVA, where the between-subjects factor was LA group and the within-subject factor was visual attribute. A significant main effect of LA group was found, $F(3,43)=20.55$, $p<.0001$, although neither the main effect of visual attribute nor the interaction was significant (visual attribute, $F(3,129)=1.14, p=.34$; interaction, $F(9,129)=1.03$, $p=.42$ ). Post hoc analyses (Tukey-Kramer, $p<.05$ ) revealed that children with a language age of $\leq 2$ years consistently named significantly fewer visual attributes than the other LA groups. Furthermore, the 3 years LA group consistently named fewer visual attributes than the older LA groups.

(3) Analysis of naming competency in relation to perceptual saliency. This cross-task analysis was performed to investigate the influence of perceptual saliency on naming ability. The preferential-matching task revealed that children fell into three different groups based on their preference behavior: no preference $(n=10)$, color preference $(n=14)$, and form preference $(n=23)$. To investigate differences in naming performance across the four visual attributes for each of the three preference groups, three repeated-measures ANOVAs were conducted. Results showed that the main effect of visual attribute was not significant for any of the preference groups (no preference group, $F(3,27)=1.34, p=.28$; colorpreference group, $F(3,39)=1.79, p=.17$; form-preference group, $F(3,66)=$ $.95, p=.43)$. This indicates that the four visual attributes were named to the same extent by each of the preference groups.

\section{DISCUSSION}

An established literature has suggested that children experience difficulties in acquiring conceptual representations of color, as evidenced by their tardy and erratic ability to name colors relative to everyday objects (see Introduction). We 
suggested that comparing the development of color cognition to that of familiar objects is not appropriate because color is an abstract object attribute and lacks the functional significance that is thought to define objects ( $\mathrm{Au} \&$ Markman, 1987; Baldwin, 1989; Macario, 1991; Soja, 1994). The important question of whether there is a selective delay in the conceptualization of color can only be addressed if the development of color cognition (recognition and naming) is compared with that of other abstract primary visual attributes that receive specialized processing by the prestriate cortex. In this study we sought to address this issue by comparing the recognition, perceptual saliency, and accurate naming of four abstract primary perceptual attributes, namely, color, motion, size, and abstract forms. In addition, we investigated whether perceptual saliency is an important factor in establishing conceptual representations of abstract visual attributes, by comparing children's performance across the preferential-matching and attribute naming tasks.

Overall, this study addresses three important issues regarding the investigation of visual cognitive development, each of which will be discussed in turn below. First, we raise a new methodological issue, that is, the use of language age rather than chronological age as a metric for studies investigating the development of visual cognition. Second, we discuss the empirical results of comparing color to that of other abstract visual attributes on tasks of recognition, preferential matching, and accurate naming. Third, we consider the implications of our results for claims that color presents a special case for conceptualization.

As tasks used to assess conceptual development (recognition and naming) are generally related to general language functioning (Johnson, 1977) we used language age rather than chronological age as the metric on which to compare children of similar ability. The correlation between language age and chronological age in our group was significant, $r=.77, p<.0001$, suggesting that a similar pattern of results would be obtained across tasks if comparisons were based on chronological age. To assess which of these metrics was the most reliable for basing comparisons, we repeated our analyses using four chronological age groups. We found a similar overall pattern of results as with language age; however, the variance of the chronological age groups was much greater than that of the language age groups. The use of chronological age may thus contribute to the variation among previous studies in the reporting of the age by which children find color to be perceptually salient (e.g., Baldwin, 1989; Miller, 1977) and can name the four primary colors (e.g., Miller \& Johnston-Laird, 1976; Shatz et al., 1996). Furthermore, comparisons based on language age rather than chronological age should control for the sex differences that have been reported in children's color naming (e.g., Anyan \& Quillian, 1971; Bernasek \& Haude, 1993; Johnson, 1977) as the superior color naming of girls compared to boys is likely to be associated with a more general superiority in language skills commonly exhibited by girls (e.g., Maccoby, 1966; McGlone, 1980). Thus, we advocate the use of language age for basing comparisons by future studies investigating the development of visual cognition, if greater concordance between studies is to be achieved. 
The empirical findings of our comparisons of color and the three other abstract visual attributes on the tasks of recognition, preferential matching, and naming raise serious problems for studies claiming that color represents a special case for conceptualization (e.g., Bornstein, 1985a; Sandhofer \& Smith, 1999). Our preferential-matching task shows that, for children at an early stage of language development, color is perceptually more salient than the other visual attributes investigated. We found a distinct developmental trend: children that expressed no particular preference had a younger language age $(M=2: 11$ years) than those expressing a significant preference for color ( $M=3: 8$ years), who, in turn, had a younger language age than those preferring form $(M=4: 8$ years). These findings support those of previous studies (e.g., Baldwin, 1989; Brian \& Goodenough, 1929; Kagan \& Lemkin, 1961; Miller, 1977; Suchman \& Trabasso, 1966). Children of a language age of around 3 years selectively group together stimuli on the basis of color, even at the expense of matching to the other visual attributes. Interestingly, we found that no children of any age exhibited a particular preference for motion or size. These properties may be less salient than color and form because they are both relative dimensions, whereas color and form are categorically organized.

Although the age at which children express a preference for color varies across studies, from 2 years (Baldwin, 1989) to 6 years (Miller, 1977), the direction of the shift from color to form is consistent. The underlying nature of the developmental shift in the saliency of color to form has been regarded by some to reflect an awareness that object shape is more indicative of category membership than is object color (e.g., Baldwin, 1989; Landau, Smith, \& Jones, 1988). Baldwin (1989) found that, on a novel word extension task, children aged 2 years were more likely to match objects on the basis of form when the target was presented in conjunction with a novel word (e.g., "See this zom? Can you find another zom?") than in the absence of a label (e.g., "Find another one") where they frequently matched according to color. In contrast, children aged 3 years almost exclusively matched according to form under both conditions. Baldwin argued that form is a more reliable indicator of an object's category membership than color and thus early language learners begin to expect linguistic labels to refer to objects similar in form rather than those similar in color. Children may shift to making form matches from color matches when they understand that form is more important than color in guiding object-label reference.

Why children find color to be perceptually salient relative to other visual attributes during the early stages of language acquisition remains to be determined. One possible explanation may lie in a greater emphasis given by parents and caregivers to color at this particular age. However, this study has clearly shown that perceptual saliency does not appear to influence attribute conceptualization as no differences were found in the cognitive development (recognition and naming) of the four perceptual attributes. As expected, we found that children's ability to recognize and name the different visual attributes improved with increasing language skills. However, in contrast to previous studies (Bornstein, 1985a; 
Sandhofer \& Smith, 1999) we found no selective difficulty with children's recognition and naming of color compared to the other visual attributes. Children of all language ages recognized color to the same extent as size and abstract forms and were able to name color with the same degree of accuracy and consistency as each of the other visual attributes. This suggests that the development of color cognition does not differ from that of other abstract visual attributes that lack functional significance and have a similar physiological and perceptual organization. Furthermore, our finding (see naming results 3) that the different visual attributes were named to the same extent, even by children who expressed a particular preference for color, or form, or no preference, adds further support for the suggestion that perceptual saliency has little influence on the conceptualization of visual attributes.

These results are intriguing as the comparable naming of color (red and green) and abstract forms $(\mathrm{O}$ and $\mathrm{T})$ by our children contrasts with a previous study that compared color and form naming and suggested that children had particular difficulties with color. Bornstein (1985a) compared the paired-associate learning of proper nouns to three colors and three abstract forms. He found that children were significantly better at learning shape-name associations than color-name associations. The discrepancy between our findings and those of Bornstein (1985a) may be due to important differences in the methods used for assessing naming between the two studies. First, we used a confrontational naming task that required children to produce the spoken names of each stimulus attribute. In contrast, Bornstein used a paired-associate learning paradigm requiring children to form mappings between a particular stimulus attribute and a proper name. Second, we presented stimuli in pairs that were identical in every attribute other than the attribute to be named. In contrast, the stimuli used by Bornstein were presented individually and were not uniformly controlled: Bornstein used a blackcolored square, triangle, and circle to assess shape-name associations and also a circle that was colored either blue, green, or yellow to assess color-name associations. The common use of a circle in both the form and color conditions may have confused the children, initially focusing their attention on the circular shape, rather than the color on which to map the proper name. This may have impeded the learning of color-name associations but would not have affected the learning of shape-name associations, as the different shapes were colored black and this was not a color on which color-name associations were made. Third, in our study, if a child made an appropriate between-category response (for example, "big" when shown a big, fast, red, T, on a trial contrasting color) the trial was repeated to draw attention to the attribute (e.g., color) to be named. Thus, we equated the attentional demands required for naming each of the visual attributes as much as possible. Bornstein does not comment on the procedure he followed to control for this issue, but the difficulties he reported in children's ability to form color-name compared with shape-name associations may reflect differences in focusing attention on color rather than difficulties in mapping linguistic labels to conceptual representations of color. 
The comparable recognition of color and size by our children also contrasts with the results of a recent study by Sandhofer and Smith (1999), who argued that color terms are acquired via a different process to size terms and entail a unique system of multiple mappings. Sandhofer and Smith (1999) reported that 2-yearolds were significantly poorer at comprehending color relative to size, yet curiously, they found no significant difference in 2-year-olds' ability to name color and size terms accurately. In keeping with Sandhofer and Smith (1999), we also found no difference in children's ability to name color and size accurately, but unlike Sandhofer and Smith (1999), our children showed the same pattern of behavior in comprehending color and size. A crucial difference between our study and that of Sandhofer and Smith (1999) may account for the discrepancy in their results. Sandhofer and Smith (1999) contrasted six color terms with two size terms. This fundamental difference in the set sizes of the two visual attributes effectively means that the color and size tasks were not equated for difficulty. This problem is particularly apparent on the comprehension task, where the children have a 1 in $2(50 \%)$ chance of correctly answering the size question "Show me the big one?" compared to a 1 in $6(16.6 \%)$ chance of correctly answering the color question "Show me the purple one." Interestingly, on the production task, where no difference was found between naming color and size, the color and size sets were more likely to be equated, due to the limited set of color terms available to most of the children. Sandhofer and Smith noted that 12 of the 13 children responded with only one or two color terms over the course of this task. Consequently, the majority of the children in their study had the same number of color terms as size terms available for naming (i.e., up to two terms). In contrast, for all of our tasks we equated for task difficulty by comparing the same number of items for each of the four perceptual attributes. In addition, our stimuli were matched for familiarity. Interestingly, when set sizes are equated, as in our study, children appear to show no particular difficulty with the conceptualization of color relative to other abstract perceptual attributes.

Sandhofer and Smith (1999) also noted that children responded to the question "What color is this?" with a color term, but not necessarily the correct one, suggesting that they initially acquired a set of color terms without the appropriate referential mappings. As the authors did not make a similar observation for size terms they consequently argued that color term learning involves linking color words to color questions and that these unique word-word maps serve to promote the mapping of color terms to their referents. This interpretation is somewhat puzzling, however, given that Sandhofer and Smith reported no significant difference in the children's accurate naming of color and size terms. If the early linking of color words to color questions promotes color word learning, as the authors suggest, then children should have shown an advantage for naming color terms over size terms, yet they found no evidence of this. Furthermore, there is evidence that children can acquire a set of labels for other, noncolor, abstract stimuli, such as numbers, before they learn the referential meaning of these terms (e.g., Wynn, 1992). This suggests that early word-word maps are not unique to 
color and may apply to a variety of stimulus classes that are conceptually abstract, until their function is learned explicitly. We suggest that abstract form stimuli, such as numbers, may be comparable to color for preschool children, because, like color, they lack functional significance, until children have realized their role within the concept of number. The children's comparable recognition and naming of color to the letters $\mathrm{T}$ and $\mathrm{O}$ in our study suggests that letters also fall into this category of abstract form stimuli that lack functional significance. Letters only become functionally relevant during reading acquisition when children become aware of their function within words. As all of the children in our study were nonreaders, the abstract forms $\mathrm{T}$ and $\mathrm{O}$ appeared to be comparable conceptually to color. A possible reason, however, for the shift in perceptual saliency from color to form observed in the older LA groups may rest in the knowledge of these children that letters, like everyday objects, are classified by shape and not color, motion, and size.

The comparable conceptualization of color and size shown by our children supports the prediction made by Braisby and Dockrell (1999), although we provide a different reason to account for this finding than that proffered by these authors. We predicted that the cognitive development of color would be comparable to that of size, motion, and abstract forms, because these are all abstract property attributes that lack functional significance. In contrast, Braisby and Dockrell (1999) proposed that children should show similar conceptualization of color to size, as both are "vague" terms with blurred concept boundaries, yet this is an untested explanation. However, our children conceptualized the "vague" object properties color, size, and motion to the same extent as the abstract forms T and O which, according to Braisby and Dockrell's (1999) rationale, should have sharp concept boundaries. The comparable conceptualization by our children of the four visual attributes that all lack functional significance but vary in the precision of their conceptual boundaries poses a serious challenge to the explanation proposed by Braisby and Dockrell (1999).

In addition there are several methodological problems with Braisby and Dockrell's (1999) study that raises concerns about their results. First, they used adult, rather than child, word frequency counts on which to match the color and animal stimuli in the high- and low-frequency groups. Adult word frequency counts may not be a good measure of the frequency by which words occur in children's language and thus may influence the pattern of their results. Second, the terms given to denote the low frequency colors may not correspond to those used in child-centered language. For example, the low-frequency color "aquamarine" is more likely to be termed "blue-green" by children and caregivers interacting with children. Third, the distinction between the high- and low-frequency groups is not clear, as the designated high-frequency animal term "peacock" has the same frequency count as the designated low-frequency color term "pink." Fourth, three of the low-frequency colors (olive, cream, and violet) are also examples of objects so it is not clear, according to Braisby and Dockrell's (1999) argument, how these terms would be represented conceptually. 
The lack of functional significance that is associated with color may inhibit its conceptualization relative to everyday functional objects (Au \& Markman, 1987; Macario, 1991; Soja, 1994). This could lead to the observation, which has been reported repeatedly in the literature, that children have a selective difficulty acquiring color terms relative to common objects and that color presents a special case for conceptualization. However, the results of our study suggest that there is nothing special about the conceptualization of color except that it is functionally less relevant than everyday objects. When compared to other abstract visual attributes, under identical experimental conditions, children do not appear to show a selective difficulty with conceptualizing color. Rather, we suggest that the delay in color naming relative to everyday objects reflects a more general delay in children's ability to conceptualize abstract object attributes compared to those with functional relevance.

In learning about specific abstract object attributes, children have the difficult task of selectively abstracting a particular attribute from the many different attributes associated with an object, such as shape, color, and size. Previous studies have shown that when object differences are explicitly contrasted, over a period of training children begin to abstract relational similarities (e.g., Kotovsky \& Gentner, 1996). It remains an interesting possibility that the difficulties that children encounter when learning about object attributes, such as color, are not a consequence of difficulties in forming mappings between attribute labels and object attributes per se (Soja, 1994). Instead, they may reflect difficulties in abstracting one particular attribute from the many possible alternatives that belong to common objects. In our naming task, we explicitly contrasted the perceptual attribute to be named by keeping the other three attributes constant. This may have served to focus the child's attention on the particular attribute being tested, and consequently we may have reduced the attentional demands that are usually involved in the learning of attribute labels. To this extent, our naming task may not reflect how children learn attribute labels during language development. However, our naming task clearly shows that when attentional demands are reduced, it is possible to show evidence of mappings between different object attributes and attribute terms even in very young children. Future studies are required to distinguish between these two interesting possibilities.

We conclude that even though young children find color to be highly salient perceptually relative to other primary visual attributes, and use color over other attributes as a criterion for matching objects, this does not advantage their ability to conceptualize color. They recognize color to the same extent as abstract forms and size and name color to a similar degree of accuracy and consistency as other abstract primary perceptual attributes when tested under identical experimental conditions. These results are surprising in that they fail to support the historical contention that color cognition is peculiarly and selectively delayed. We suggest instead that the developmental delay in color cognition may be part of a more general delay in the cognition of abstract object attributes. 


\section{APPENDIX 1 \\ STIMULUS PAIRS USED IN THE RECOGNITION TASK}

\section{Trial}

Stimulus 1

$\mathrm{T}$, red, fast, big

$\mathrm{T}$, red, slow, big

$\mathrm{T}$, red, fast, small

$\mathrm{T}$, red, slow, small

$\mathrm{T}$, green, fast, big

$\mathrm{T}$, green, slow, big

$\mathrm{T}$, green, fast, small

$\mathrm{T}$, green, slow, small
Stimulus 2

O, green, slow, small

$\mathrm{O}$, green, fast, small

$\mathrm{O}$, green, slow, big

$\mathrm{O}$, green, fast, big

$\mathrm{O}$, red, slow, small

$\mathrm{O}$, red, fast, small

$\mathrm{O}$, red, slow, big

$\mathrm{O}$, red, fast, big

\section{APPENDIX 2 \\ STIMULI USED IN THE PREFERENTIAL-MATCHING TASK}

\begin{tabular}{llllll}
\hline \multirow{2}{*}{ Trial } & \multicolumn{3}{c}{ Target } & \multicolumn{1}{c}{ Form } & \multicolumn{2}{c}{ Color } & \multicolumn{2}{c}{ Motion } & \multicolumn{1}{c}{ Size } \\
\cline { 3 - 5 } & O, R, F, B & O, G, S, Sm & T, R, S, Sm & T, G, F, Sm & T, G, S, B \\
\hline 1 & O, R, S, B & O, G, F, Sm & T, R, F, Sm & T, G, S, Sm & T, G, F, B \\
3 & O, R, F, Sm & O, G, S, B & T, R, S, B & T, G, F, B & T, G, S, Sm \\
4 & O, R, S, Sm & O, G, F, B & T, R, F, B & T, G, S, B & T, G, F, Sm \\
5 & O, G, F, B & O, R, S, Sm & T, G, S, Sm & T, R, F, Sm & T, R, S, B \\
6 & O, G, S, B & O, R, F, Sm & T, G, F, Sm & T, R, S, Sm & T, R, F, B \\
7 & O, G, F, Sm & O, R, S, B & T, G, S, B & T, R, F, B & T, R, S, Sm \\
8 & O, G, S, Sm & O, R, F, B & T, G, F, B & T, R, S, B & T, R, F, Sm \\
9 & T, R, F, B & T, G, S, Sm & O, R, S, Sm & O, G, F, Sm & O, G, S, B \\
10 & T, R, S, B & T, G, F, Sm & O, R, F, Sm & O, G, S, Sm & O, G, F, B \\
11 & T, R, F, Sm & T, G, S, B & O, R, S, B & O, G, F, B & O, G, S, Sm \\
12 & T, R, S, Sm & T, G, F, B & O, R, F, B & O, G, S, B & O, G, F, Sm \\
13 & T, G, F, B & T, R, S, Sm & O, G, S, Sm & O, R, F, Sm & O, R, S, B \\
14 & T, G, S, B & T, R, F, Sm & O, G, F, Sm & O, R, S, Sm & O, R, F, B \\
15 & T, G, F, Sm & T, R, S, B & O, G, S, B & O, R, F, B & O, R, S, Sm \\
16 & T, G, S, Sm & T, R, F, B & O, G, F, B & O, R, S, B & O, R, F, Sm \\
\hline
\end{tabular}

Note. Stimuli for each of the four visual attributes are abbreviated as follows: form, $\mathrm{O}$ and T; color, red $(\mathrm{R})$ and green $(\mathrm{G})$; motion, fast $(\mathrm{F})$ and slow $(\mathrm{S})$; and size, big $(\mathrm{B})$ and small $(\mathrm{Sm})$. 


\section{APPENDIX 3 \\ STIMULI USED IN THE CONFRONTATIONAL NAMING TASK}

\begin{tabular}{lllll}
\hline \multirow{2}{*}{ Stimulus pair } & \multicolumn{2}{c}{ Visual attribute } \\
\cline { 2 - 5 } & \multicolumn{1}{c}{ Form } & Color & Motion & Size \\
\hline \multirow{2}{*}{2} & O, R, F, B & O, R, F, B & O, R, F, B & O, R, F, B \\
& T, R, F, B & O, G, F, B & O, R, S, B & O, R, F, Sm \\
3 & O, R, S, B & O, R, S, B & O, G, F, B & O, R, S, B \\
& T, R, S, B & O, G, S, B & O, G, S, B & O, R, S, Sm \\
4 & O, R, F, Sm & O, R, F, Sm & O, R, F, Sm & O, G, F, B \\
& T, R, F, Sm & O, G, F, Sm & O, R, S, Sm & O, G, F, Sm \\
5 & O, R, S, Sm & O, R, S, Sm & O, G, F, Sm & O, G, S, B \\
& T, R, S, Sm & O, G, S, Sm & O, G, S, Sm & O, G, S, Sm \\
6 & O, G, F, B & T, R, F, B & T, R, F, B & T, R, F, B \\
& T, G, F, B & T, G, F, B & T, R, S, B & T, R, F, Sm \\
7 & O, G, S, B & T, R, S, B & T, G, F, B & T, R, S, B \\
& T, G, S, B & T, G, S, B & T, G, S, B & T, R, S, Sm \\
& O, G, F, Sm & T, R, F, Sm & T, R, F, Sm & T, G, F, B \\
& T, G, F, Sm & T, G, F, Sm & T, R, S, Sm & T, G, F, Sm \\
& O, G, S, Sm & T, R, S, Sm & T, G, F, Sm & T, G, S, B \\
\hline & T, G, S, Sm & T, G, S, Sm & T, G, S, Sm & T, G, S, Sm \\
\hline
\end{tabular}

Note. Stimuli for each of the four visual attributes are abbreviated as follows: Form, $\mathrm{O}$ and T; color, red $(\mathrm{R})$ and green $(\mathrm{G})$; motion, fast $(\mathrm{F})$ and slow $(\mathrm{S})$; and size, big $(\mathrm{B})$ and small $(\mathrm{Sm})$.

\section{REFERENCES}

Allen, D., Banks, M. S., \& Norcia, A. M. (1993). Does chromatic sensitivity develop more slowly than luminance sensitivity? Vision Research, 33, 2553-2562.

Andrick, G., \& Tager-Flusberg, H. (1986). The acquisition of color terms. Journal of Child Language, 13, 119-134.

Anyan, W. R., \& Quillian, W. W. (1971). The naming of primary colors by children. Child Development, 42, 1629-1632.

Au, T. K., \& Markman, E. M. (1987). Acquiring word meanings via linguistic contrast. Cognitive Development, 2, 217-236.

Baldwin, D. (1989). Priorities in children's expectations about object label reference: Form over color. Child Development, 60, 1291-1306.

Baldwin, J. M. (1893). A new method of child study. Science, 21, 213-214.

Bartlett, E. J. (1978). The acquisition of the meaning of color terms: a study of lexical development. In R. Campbell \& P. Smith (Eds), Recent advances in the psychology of language. New York: Plenum.

Bateman, W. G. (1915). The naming of colors by children: The Binet test. Pedagogical Seminary, 22, 469-486.

Bernasek, J. L., \& Haude, R. H. (1993). Color-naming and color-matching by preschool children as a function of visual field and sex. Perceptual and Motor Skills, 77, 739-747.

Binet, A., \& Simon, T. (1908/1916). [The development of intelligence in the child] (E. S. Kiffe, Trans.). In H. H. Goddard (Ed.), The development of intelligence in children. Baltimore: Williams \& Wilkins.

Bornstein, M. H. (1985a). Color-name versus shape-name learning in young children. Journal of Child Language, 12, 387-393. 
Bornstein, M. H. (1985b). On the development of color naming in young children: Data and theory. Brain and Language, 26, 72-93.

Bornstein, M. H., Kessen, W., \& Weiskopf, S. (1976). Color vision and hue categorization in young human infants. Journal of Experimental Psychology: Human Perception and Performance, 2, $115-129$.

Braisby, N., \& Dockrell, J. (1999). Why is colour naming difficult? Journal of Child Language, 26, 23-47.

Brian, C. R., \& Goodenough, F. L. (1929). The relative potency of color and form perception at various ages. Journal of Experimental Psychology, 12, 197-213.

Carey, S. (1978). The child as a word learner. In M. Halle, J. Bresnan, \& G. Miller (Eds.), Linguistic theory and psychological reality. Cambridge, MA: MIT Press.

Cruse, D. A. (1977). A note on the learning of color names. Journal of Child Language, 4, 305-311.

Davidoff, J. B. (1991). Cognition through color. Cambridge, MA: MIT Press.

De Yeo, E. A., \& Van Essen, D. C. (1988). Concurrent processing streams in monkey visual cortex. Trends in Neuroscience, 11, 219-226.

Hadjikhani, N., Liu, A. K., Anders, M. D., Cavanagh, P., \& Tootell, R. B. H. (1998). Retinotopy and color sensitivity in human visual cortical area V8. Nature Neuroscience, 1, 235-241.

Heider, E. R. (1971). "Focal" color areas and the development of color names. Developmental Psychology, 4, 447-455.

Itskowitz, R., Strauss, H., \& Gross, Y. (1987). Differential use of dimensions in similarity and preference judgements among 4- to 6-year-olds. Perceptual and Motor Skills, 65, 147-156.

Johnson, E. G. (1977). The development of color knowledge in preschool children. Child Development, 48, 308-311.

Kagan, J., \& Lemkin, J. (1961). Form, color and size in children's conceptual behavior. Child Development, 32, 25-28.

Kotovsky, L., \& Gentner, D. (1996). Comparison and categorization in the development of relational similarity. Child Development, 67, 2797-2822.

Landau, B., Smith, L. B., \& Jones, S. S. (1988). The importance of shape in early lexical learning. Cognitive Development, 3, 299-321.

Livingstone, M., \& Hubel, D. (1988). Segregation of form, color, movement and depth: Anatomy, physiology, and perception. Science, 240, 740-749.

Macario, J. F. (1991). Young children's use of color in classification: Foods and canonically colored objects. Cognitive Development, 6, 17-46.

Maccoby, E. E. (1966). The development of sex differences. Stanford: Stanford Univ. Press.

Markman, E. M. (1989). Categorization and naming in children: Problems of induction. Cambridge, MA: MIT Press.

Maurer, D., \& Adams, R. J. (1987). Emergence of the ability to discriminate blue from gray at one month of age. Journal of Experimental Child Psychology, 44, 147-156.

McGlone, J. (1980). Sex differences in human brain asymmetry: A critical survey. Behavioral and Brain Sciences, 3, 215-263.

Melkman, R., Koriat, A., \& Pardo, K. (1976). Preference for color and form in preschoolers as related to color and form differentiation. Child Development, 47, 1045-1050.

Mervis, C. B., Catlin, J., \& Rosch, E. (1975). Development of the structure of color categories. Developmental Psychology, 11, 54-60.

Miller, D. (1977). Color/form matching as indicator of cognitive reorganization in kindergarten children. The Journal of Genetic Psychology, 130, 271-278.

Miller, G. A., \& Johnston-Laird, P. N. (1976). Language and perception. Cambridge, MA: Harvard Univ. Press.

Modreski, R. A., \& Goss, A. E. (1969). Young children's initial and changed names form-color stimuli. Journal of Experimental Child Psychology, 8, 402-409.

Nagel, W. A. (1906). Observations on the color-sense of a child. Journal of Comparative Neurology and Psychology, 16, 217-230. 
Nelson, K. (1991). Concepts of meaning in language development. In N. A. Krasnegor \& D. M. Rumbaugh (Eds.), Biological and behavioral determinants of language development (pp. (89-115). Hillsdale, NJ: Erlbaum.

Rice, M. (1980). Cognition to language: Categories, word meanings, and training. Baltimore: University Park Press.

Sandhofer, C. M., \& Smith, L. B. (1999). Learning color words involves learning a system of mappings. Developmental Psychology, 35, 668-679.

Shatz, M., Behrend, D., Gelman, S. A., \& Ebeling, K. S. (1996). Color term knowledge in two-yearolds: evidence for early competence. Journal of Child Language, 23, 177-199.

Soja, N. N. (1994). Young children's concept of color and its relation to the acquisition of color words. Child Development, 65, 918-937.

Suchman, R. G., \& Trabasso, T. (1966). Color and form preference in young children. Journal of Experimental Child Psychology, 3, 177-187.

Teller, D. Y. (1998). Spatial and temporal aspects of color vision. Vision Research, 38, 3275-3282.

Teller, D. Y., \& Bornstein, M. H. (1985). Color vision and color perception in infancy. In L. B. Cohen \& P. Salapatek (Eds.), Handbook of infant perception. New York: Academic Press.

Waxman, S. R., \& Markow, D. B. (1998). Object properties and object kind: twenty-one-month-old infants' extension of novel adjectives. Child Development, 69, 1313-1329.

Wynn, K. (1992). Children's acquisition of the number words and the counting system. Cognitive Psychology, 24, 220-251.

Zimmerman, I. L., Steiner, V. G., \& Pond, R. E. (1992). The Preschool Language Scale-3. San Antonio, TX: Psychol. Corp.

Received August 4, 2000; revised February 12, 2001; published online June 7, 2001 